\title{
Differing associations for sport versus occupational physical activity and cardiovascular risk
}

\author{
Marco Mario Ferrario, ${ }^{1,2,3}$ Mattia Roncaioli, ${ }^{3}$ Giovanni Veronesi, ${ }^{1}$ \\ Andreas Holtermann, ${ }^{4,5}$ Els Clays, ${ }^{6}$ Rossana Borchini, ${ }^{2}$ Marco Cavicchiolo, ${ }^{1}$ \\ Guido Grassi, ${ }^{7,8}$ Giancarlo Cesana, ${ }^{7}$ on behalf of The Cohorts Collaborative Study in \\ Northern Italy (CCSNI) Research Group
}

\begin{abstract}
- Additional material is published online only. To view please visit the journal online (http://dx.doi.org/10.1136/ heartjnl-2017-312594).
\end{abstract}

For numbered affiliations see end of article.

\section{Correspondence to}

Professor Marco Mario Ferrario, Department of Medicine and Surgery, Research Centre on Epidemiology and Preventive Medicine, University of Insubria at Varese, Varese 21100, Italy; marco.ferrario@uninsubria.it

Received 19 October 2017 Revised 28 December 2017 Accepted 5 January 2018

Published Online First 9 February 2018
Linked

- http://dx.doi.org/10.1136/ heartjnl-2017-312891

Check for updates

To cite: Ferrario MM, Roncaioli M, Veronesi $G$, et al. Heart 2018;104:1165-1172.

\section{ABSTRACT}

Objectives We investigate the independent and interacting long-term associations of occupational physical activity (OPA) and sport physical activity (SpPA) with the incidence of coronary heart disease (CHD) and cardiovascular diseases (CVD; CHD plus ischaemic stroke) in North Italian male workers.

Methods 3574 employed men aged 25-64 years, free of CVD at baseline, recruited in three population-based and one factory-based cohorts, were included in the analysis. The Baecke Questionnaire was used to assess OPA and SpPA in 'minutes per week' of moderate or vigorous PA. We estimated the associations between different domains of PA and the endpoints, adjusting for major CVD risk factors, using Cox models.

Results During a median follow-up of 14 years, 135 and 174 first CHD and CVD events, fatal and non-fatal, occurred. Compared with the intermediate OPA tertile, the HRs for CHD among low and high OPA workers were $1.66(95 \% \mathrm{Cl} 1.06$ to 2.59) and 1.18 (0.72 to 1.94), respectively ( $P$ value $=0.07)$. Decreasing trends in $C H D$ and CVD rates across increasing levels of SPPA were also found, with an HR for CVD of 0.68 (0.46 to 0.98) for intermediate/recommended SpPA compared with poor SpPA. We also found a statistically significant SpPA-OPA interaction, and the protective effect of SpPA was only found among sedentary workers, for both endpoints. Conversely, high OPA workers with intermediate/ recommended SpPA levels had increased CHD and CVD rates compared with the poor SpPA category.

Conclusions Our results provide further evidence on the health paradox of OPA, with higher CVD rates among workers with intense PA at work. Moreover, the protective effect on CVDs of SpPA is prominent in sedentary workers, but it attenuates and even reverses in moderate and strenuous OPA workers.

\section{INTRODUCTION}

The 2015 Global Burden of Disease Study estimated that 15.2 million people died worldwide from coronary heart disease (CHD) and stroke. ${ }^{1}$ In Europe alone, CHD accounts for 1.8 million deaths annually. $^{2}$ Physical activity (PA) is known as an important modifiable protective factor for the development of CHD and as such it has been included as an integral component of more updated cardiovascular prevention guidelines. ${ }^{3}$ Since 2010, the American Heart Association (AHA) recommends adults to perform at least $150 \mathrm{~min}$ per week of moderate-intensity activity or at least $75 \mathrm{~min}$ per week of vigorous-intensity activity, or at least $150 \mathrm{~min}$ per week of moderate and vigorous-intensity activities combined. These recommendations were integrated into the WHO global recommendations on $\mathrm{PA},{ }^{5}$ and allow classifying adults into three classes: poor, including those who practice none PA; intermediate, including those who practice PA without reaching the recommended minutes per week; and ideal, including those who practice PA for the recommended minutes per week. ${ }^{6}$

While most studies consistently indicated that high levels of leisure time physical activity (LTPA) decrease the risk of cardiovascular disease (CVD), ${ }^{7-22}$ the relationship between occupational PA (OPA) and CVD has shown contradictory results. A few studies have reported both intermediate and high levels of OPA as protective factors for CHD and stroke ${ }^{91516}$ or intermediate levels of OPA only as a protective factor for CVD mortality and incidence. ${ }^{1113}$ Conversely, other studies indicated that moderate and high levels of OPA increased the risk of major cardiovascular events. ${ }^{14} 1721$ Finally, other studies did not find any association. ${ }^{12}{ }^{19}$ Some studies have also estimated the interplay between LTPA and OPA on the CVD risk ${ }^{15}{ }^{17-22}$ without univocal results, in particular concerning the effect of SpPA within groups exposed to intense OPA.

The aim of this study is to investigate the independent and interacting associations between OPA and SpPA categories, the latter based on AHA recommendations, and the incidence of coronary events in North Italian working men, over a follow-up of a median of 14 years.

\section{MATERIAL AND METHODS \\ Study samples}

This study is a pooled analysis of three population-based (the Multinational Monitoring of Trends and Determinants in Cardiovascular Disease (MONICA)—Brianza surveys II and III and the Pressioni Arteriose Monitorate e Loro Associazioni (PAMELA)-Study) and one factory-based (the Study of Employed in the Municipality of Milan (SEMM)—study) prospective cohorts of men aged 25 to 64 years, working as salaried employees (executive, white and bluecollar workers). ${ }^{2324}$ No written informed consent 
Table 1 Baseline demographic characteristics and CVD risk factors according to occupational PA categories

\begin{tabular}{|c|c|c|c|c|}
\hline & \multicolumn{3}{|l|}{ Occupational PA } & \multirow[b]{2}{*}{$P$ value } \\
\hline & Low $(n=1343)$ & Intermediate $(n=1227)$ & High $(n=1104)$ & \\
\hline Age, years (SD) & $42.8(9.1)$ & $39.9(8.9)$ & $39.6(9.2)$ & $<0.0001$ \\
\hline \multicolumn{5}{|l|}{ Cohort type, $\%$} \\
\hline Population-based & 42.1 & 33.0 & 43.0 & \multirow[t]{2}{*}{$<0.0001$} \\
\hline Factory-based & 57.9 & 67.0 & 57.0 & \\
\hline \multicolumn{5}{|l|}{ Occupational class, \% } \\
\hline Executives & 20.7 & 8.3 & 5.6 & \multirow[t]{2}{*}{$<0.0001$} \\
\hline Non-manual and manual workers & 79.3 & 91.7 & 94.4 & \\
\hline \multicolumn{5}{|l|}{ Educational class* $\%$} \\
\hline Low & 22.5 & 35.8 & 55.8 & \multirow[t]{3}{*}{$<0.0001$} \\
\hline Intermediate & 32.2 & 36.1 & 27.7 & \\
\hline High & 45.4 & 28.1 & 16.5 & \\
\hline Body mass index, $\mathrm{kg} / \mathrm{m}^{2}$ & 26.2 & 26.4 & 26.5 & 0.13 \\
\hline Total cholesterol, mg/dL & 217.3 & 217.0 & 214.4 & 0.15 \\
\hline HDL cholesterol, mg/dL & 48.2 & 49.7 & 49.7 & 0.004 \\
\hline Total cholesterol/HDL cholesterol & 4.80 & 4.66 & 4.60 & 0.001 \\
\hline Systolic BP, mm Hg & 145.4 & 145.1 & 144.5 & 0.56 \\
\hline Diastolic BP, mm Hg & 99.1 & 99.0 & 97.8 & 0.14 \\
\hline Antihypertensive treatment, \% & 6.3 & 5.0 & 5.2 & 0.32 \\
\hline Hypertensiont, \% & 63.5 & 63.5 & 62.5 & 0.90 \\
\hline Current smoking, \% & 34.5 & 40.1 & 41.4 & 0.001 \\
\hline Diabetes, $\%$ & 2.82 & 1.31 & 2.93 & 0.03 \\
\hline \multicolumn{5}{|l|}{ Alcohol intake* $\%$} \\
\hline None & 36.8 & 34.3 & 32.7 & \multirow[t]{3}{*}{0.003} \\
\hline Moderate (<50 gr/die) & 56.9 & 58.6 & 57.4 & \\
\hline High (>50 gr/die) & 6.3 & 7.1 & 9.9 & \\
\hline \multicolumn{5}{|l|}{ Sport PA* $\%$} \\
\hline Poor & 64.0 & 69.1 & 77.7 & \multirow[t]{3}{*}{$<0.0001$} \\
\hline Intermediate & 21.7 & 18.4 & 13.4 & \\
\hline Recommended & 14.3 & 12.5 & 8.9 & \\
\hline
\end{tabular}

signed by participants was required at time of recruitment. The study cohorts summed up to 3995 men who were free of CVD and currently employed at baseline. Women were not included in these analyses due to the low number of events during follow-up: 37 and 27 first coronary and ischaemic stroke events, respectively. A total of 421 men (10.5\%) were excluded from the analysis due to missing information on CVD risk factors $(n=175)$ or on PA $(n=246)$. The final sample size consisted of 3574 male workers.

\section{Baseline risk factors assessment and follow-up procedures}

Information on adopted procedures to measure CVD risk factors at baseline and to detect endpoints has been published ${ }^{23-25}$; we summarise here major methodological aspects. The MONICA, PAMELA and SEMM baseline risk factors assessments were carried out by a unique team between 1989 and 1996 . The overall participation rate was not $<65 \%$, with small variations among the four cohorts. Cardiovascular risk factors were collected adhering to the standardised procedures and quality standards of the WHO-MONICA Project (http://www.thl.fi/publications/monica/manual/index.htm). In the PAMELA and in the SEMM studies, risk factors were measured based on MONICA-like procedures (see online supplementary material).
PA was measured using the Baecke Questionnaire, ${ }^{26}$ a known reliable and valid tool, ${ }^{27}$ to assess habitual PA. The questionnaire consists of 16 items from which an OPA score (eight items) and an SpPA index (four items) were derived. The OPA items investigated participant's main occupation; his/her selfrating of the work's vigour as compared with workers of the same age; the frequency of sitting, standing, walking, lifting and sweating at work; and the frequency of fatigue after work. The OPA score was categorised in tertiles, with cut-offs at 2.5 and 3.125 points (on a scale ranging from 1 to 5). Based on the method suggested by Atherosclerosis Risk in Communities (ARIC) investigators, ${ }^{10} \mathrm{SpPA}$ items were converted in 'minutes per week' of moderate or vigorous PA based on metabolic equivalent of task, ${ }^{28}$ over the number of months annually performing the activities. The 'minutes per week' variable was then categorised into SpPA categories, according to AHA CVD health guidelines $^{6}$ : poor SpPA ( $0 \mathrm{~min} /$ week of activity); intermediate SpPA (1-149 min/week moderate or 1-74 min/week vigorous or 1-149 min/week moderate plus vigorous activity); and recommended SpPA $(\geq 150 \mathrm{~min} /$ week moderate or $\geq 75 \mathrm{~min} /$ week vigorous or $\geq 150$ moderate plus vigorous activity).

The same follow-up procedures were adopted for all cohorts, and people were followed until the end of 2008. The study endpoints were (1) a first acute coronary event as myocardial 
Table 2 Baseline demographic characteristics and CVD risk factors according to sport PA categories

\begin{tabular}{|c|c|c|c|c|}
\hline & \multicolumn{3}{|l|}{ Sport PA } & \multirow[b]{2}{*}{$P$ value } \\
\hline & Poor $(n=2231)$ & Intermediate $(n=737)$ & Recommended ( $n=606)$ & \\
\hline Age, years (SD) & $42.8(9.0)$ & $38.7(8.7)$ & $36.8(8.2)$ & $<0.0001$ \\
\hline \multicolumn{5}{|l|}{ Cohort type, \% } \\
\hline Population-based & 41.3 & 40.7 & 31.7 & \multirow[t]{2}{*}{$<0.0001$} \\
\hline Factory-based & 58.7 & 59.3 & 68.3 & \\
\hline \multicolumn{5}{|l|}{ Occupational class, $\%$} \\
\hline Executives & 11.4 & 16.0 & 10.1 & \multirow[t]{2}{*}{$<0.01$} \\
\hline Non-manual and manual workers & 88.6 & 84.0 & 89.9 & \\
\hline \multicolumn{5}{|l|}{ Educational class ${ }^{*} \%$} \\
\hline Low & 40.1 & 26.3 & 29.8 & \multirow[t]{3}{*}{$<0.0001$} \\
\hline Intermediate & 31.1 & 32.2 & 36.6 & \\
\hline High & 28.7 & 41.5 & 33.6 & \\
\hline Body mass index, $\mathrm{kg} / \mathrm{m}^{2}$ & 26.5 & 26.0 & 26.1 & $<0.01$ \\
\hline Total cholesterol, mg/dL & 217.1 & 217.8 & 210.9 & $<0.01$ \\
\hline HDL cholesterol, mg/dL & 48.5 & 50.0 & 51.0 & $<0.0001$ \\
\hline Total cholesterol/HDL cholesterol & 4.77 & 4.64 & 4.42 & $<0.0001$ \\
\hline Systolic BP, mm Hg & 144.8 & 144.9 & 146.3 & 0.27 \\
\hline Diastolic BP, mm Hg & 98.4 & 98.8 & 99.9 & 0.15 \\
\hline Antihypertensive treatment, \% & 6.0 & 4.2 & 5.3 & 0.23 \\
\hline Hypertensiont, \% & 62.9 & 62.5 & 65.2 & 0.65 \\
\hline Current smoking, \% & 43.2 & 32.8 & 27.3 & $<0.0001$ \\
\hline Diabetes, $\%$ & 2.54 & 1.94 & 2.32 & 0.71 \\
\hline \multicolumn{5}{|l|}{ Alcohol intake* $\%$} \\
\hline None & 34.4 & 35.4 & 36.5 & \multirow[t]{3}{*}{0.15} \\
\hline Moderate (<50 gr/die) & 57.4 & 57.9 & 58.2 & \\
\hline High (>50 gr/die) & 8.3 & 6.7 & 5.3 & \\
\hline \multicolumn{5}{|l|}{ Occupational PA* $\%$} \\
\hline Low & 37.6 & 48.4 & 47.1 & \multirow[t]{3}{*}{$<0.0001$} \\
\hline Intermediate & 30.0 & 30.5 & 31.3 & \\
\hline High & 32.4 & 21.1 & 21.7 & \\
\hline
\end{tabular}

Men aged 25-64 years, free of CVD and current employed at baseline ( $n=3574)$. Age-adjusted mean (prevalence) at the age of 45 years, from linear (logistic) models.

*Prevalence estimated from a generalised logits model.

tSystolic BP $\geq 140 \mathrm{~mm} \mathrm{Hg}$ or diastolic $B P \geq 90 \mathrm{~mm} \mathrm{Hg}$ or under anti-hypertensive treatment.

BP, blood pressure; CVD, cardiovascular disease; HDL, high-density lipoprotein; PA, physical activity.

infarction, acute coronary syndrome or coronary revascularisation whichever occurred first; and (2) a first major cardiovascular event, including in addition a first ischaemic stroke or carotid endarterectomy. Loss-to-follow-up rate was $<4 \%$, with no differences across study cohorts.

\section{Statistical analysis}

We estimated the age-adjusted mean (prevalence) of major CVD risk factors by OPA and SpPA categories from linear (logistic) regression models, and tested differences among groups using the Wald $\chi^{2}$ test. A generalised logits model was used whenever the CVD risk factor had more than two levels. To estimate the association of OPA and SpPA categories with the incidence of cardiovascular events, we used Cox proportional hazards models, including both SpPA and OPA indexes and (1) age, cohort type (population vs factory-based), educational level (low, intermediate and high); and (2) age, cohort type, educational level, total and high-density lipoprotein (HDL) cholesterol, systolic blood pressure, current smoking, diabetes, alcohol intake and body mass index (BMI). Since the association between SpPA categories and CVD risk was linear in the ARIC study ${ }^{10}$ as well as in our preliminary analysis on event rates, we tested for statistical trend by additionally running each model with SpPA treated as a continuous variable. To investigate the effect of the length of follow-up time on CVD risk, we plotted Kaplan-Meier survival curves across OPA and SpPA categories. Finally, we explored the protective role of SpPA among workers engaged in different levels of OPA by adding relevant SpPA-OPA interaction terms in age and CVD risk factor-adjusted Cox regression models, and formally testing the presence of additive interaction on the $\log (\mathrm{HR})$ scale with a Wald $\chi^{2}$ test. The analyses were performed using the Statistical Analysis System (V.9.4 release; SAS Institute, Cary, North Carolina, USA), while figures were drawn using R (V.3.1.3, 2015, The R Foundation for Statistical Computing, Wien, Austria).

\section{RESULTS}

Among the included 3574 men, in a median follow-up time of 14 years (IQR 12.9-15.9 years) 135 first fatal and non-fatal CHD events, and 174 CVD events, occurred. Age-adjusted demographic characteristics and CVD risk factor levels at baseline for OPA and SpPA categories are shown in tables 1 and 2, respectively. Workers in high OPA were younger, less educated, with higher prevalence of current smokers and more than $50 \mathrm{gr}$ per day alcohol consumers. People in the recommended SpPA category showed a better CVD risk factor profile, with lower mean levels of total cholesterol and BMI, higher median levels of HDL cholesterol and less current smokers. 
Table 3 Age and multiple risk factor-adjusted HRs (with 95\% Cls) for the incidence of CHD and CVD events across OPA and SpPA categories

\begin{tabular}{|c|c|c|c|c|c|c|c|}
\hline & $\mathrm{N}$ & Ev. no & Rate & Model 1 & Model 2 & Model 1 & Model 2 \\
\hline \multicolumn{8}{|l|}{ CHD events ( $n=135)$} \\
\hline \multicolumn{8}{|l|}{ OPA } \\
\hline Low & 1343 & 69 & 3.20 & 1.66 (1.06 to 2.59$)$ & 1.61 (1.02 to 2.52 ) & & \\
\hline Intermediate & 1127 & 29 & 2.07 & REF & REF & & \\
\hline High & 1104 & 37 & 2.61 & 1.18 (0.72 to 1.94$)$ & 1.26 (0.76 to 2.08$)$ & & \\
\hline P value* & & & & 0.07 & 0.11 & & \\
\hline \multicolumn{8}{|l|}{ SpPA } \\
\hline Poor & 2231 & 105 & 2.97 & REF & REF & REF & REF \\
\hline Intermediate & 737 & 20 & 2.46 & 0.81 (0.50 to 1.32 ) & 0.87 (0.53 to 1.42 ) & & \\
\hline Recommended & 606 & 10 & 1.78 & 0.58 (0.30 to 1.12$)$ & 0.76 (0.39 to 1.48$)$ & 0.72 (0.47 to 1.09 ) & 0.83 (0.54 to 1.27$)$ \\
\hline$P$ valuet & & & & 0.08 & 0.36 & 0.12 & 0.39 \\
\hline \multicolumn{8}{|l|}{ CVD events ( $n=174)$} \\
\hline \multicolumn{8}{|l|}{ OPA } \\
\hline Low & 1343 & 82 & 3.74 & 1.54 (1.04 to 2.29$)$ & 1.50 (1.01 to 2.23 ) & & \\
\hline Intermediate & 1127 & 39 & 2.77 & REF & REF & & \\
\hline High & 1104 & 53 & 3.77 & 1.18 (0.77 to 1.79$)$ & 1.20 (0.78 to 1.84$)$ & & \\
\hline Pvalue* & & & & 0.13 & 0.12 & & \\
\hline \multicolumn{8}{|l|}{ SpPA } \\
\hline Poor & 2231 & 139 & 3.95 & REF & REF & REF & REF \\
\hline Intermediate & 737 & 23 & 2.94 & 0.76 (0.49 to 1.20$)$ & 0.81 (0.51 to 1.27 ) & & \\
\hline Recommended & 606 & 12 & 2.24 & 0.58 (0.32 to 1.05$)$ & 0.72 (0.39 to 1.32$)$ & 0.68 (0.46 to 0.98$)$ & 0.77 (0.53 to 1.14$)$ \\
\hline P valuet & & & & 0.04 & 0.19 & 0.04 & 0.19 \\
\hline
\end{tabular}

Men aged 25-64 years, free of CVD and currently employed at baseline ( $n=3574)$. Rates (x1000 person-years) are age-adjusted and estimated at the sample mean age.

Model 1: age, cohort, educational level, OPA and SpPA.

Model 2: model 1 plus body mass index, total cholesterol, HDL cholesterol, systolic BP, current smoking, diabetes and alcohol intake.

*Wald heterogeneity $\chi^{2}$ test $(2 \mathrm{df})$.

tWald trend $\chi^{2}$ test $(1 \mathrm{df})$.

CHD, coronary heart disease; CVD, cardiovascular disease; HDL, high- density lipoprotein; OPA, occupational physical activity; SpPA, sport physical activity .

The age, cohort and education-adjusted HRs and 95\% CIs for the incidence of major CHD and CVD events across OPA and SpPA categories are shown in table 3 . As these rates and HRs are mutually adjusted for the other type of PA, their estimates of the associations between two exposures (OPA and SpPA) with the endpoints are reciprocally independent. Keeping the intermediate OPA as the reference category, low OPA showed a significant higher age and educational class-adjusted CHD risk with HRs of 1.66 (95\% CI 1.06 to 2.59) and high OPA an increased risk of 1.18 (0.72 to 1.94$)$, but not reaching statistical significance. These results were not substantially modified when adjusting for other CHD risk factors, and were confirmed, with minor attenuations of HRs, for CVD as the endpoint.

Progressively decreased CHD risks were detected in the intermediate and the recommended SpPA categories, in comparison to the poor SpPA category $\left(\chi^{2}\right.$ test for trend borderline statically significant at 0.08 ), with an overall reduction of $28 \%$ in $\mathrm{CHD}$ risk when considering the two categories combined. Here, the adjustments for other CVD risk factors reduced the associations, as most of them can be considered as direct or indirect mediators. The results were consistent for the CVD endpoint, with the notable observation that the reduced risk across SpPA categories turned statistically significant $\left(\chi^{2}\right.$ test for trend, $\left.\mathrm{P}=0.04\right)$ and the HR when considering the two intermediate and recommended categories combined was 0.68 (0.46 to 0.98$)$.

The Kaplan-Meyer survival curves show that the reduced CHD (figure 1A) and CVD (figure 1B) risks of intermediate and recommended SpPA in comparison to the poor SpPA category started from the very beginning of the follow-up period, and increased progressively. Similarly, the excessive risk of the low OPA category in comparison to the two other categories was also clearly detectable for both endpoints (figure 1C,D), but with the notable exception that the risks for poor OPA started to reveal later in the follow-up period ( $3-5$ years after baseline). This latency time may explain some of the discrepancies of the results in the literature, when the follow-up time is short.

Table 4 reports the risk factor-adjusted HRs (with 95\% CI) for SpPA in different OPA categories, and the formal test for SpPA-OPA interaction on CHD and CVD risks. The analyses were carried out combining the intermediate and recommend SpPA categories to be able to reach sufficient statistical power to test for statistical interaction. In low OPA workers, characterised mainly by sedentary work activities, prominent protective effects of recommended or intermediate SpPA were seen, with consistent reduced HRs of 0.45 (95\% CI 0.24 to 0.87 ) and 0.45 (95\% CI 0.25 to 0.82 ) for CHD and CVD, respectively, for the two active SpPA categories combined. These decreased HRs for higher levels of SpPA disappeared in the intermediate OPA category, and even reversed in the high OPA categories with values of 1.84 (0.88 to 3.87 ) for CHD and 1.66 (0.87 to 3.14) for CVD. The heterogeneity tests and the interaction tests were statically significant at an $\alpha$ level of 0.05 , for both endpoints. The detrimental effect of SpPA among high OPA workers was even more pronounced when recommended SpPA levels were met (online supplementary table S1) and when taking into account commuting PA (walking or cycling to work for more than $30 \mathrm{~min} /$ day; online supplementary table S2).

\section{DISCUSSION}

We found long-lasting (over a median 14 years of follow-up) and prominent protective effects of performing SpPA on CHD and 
A

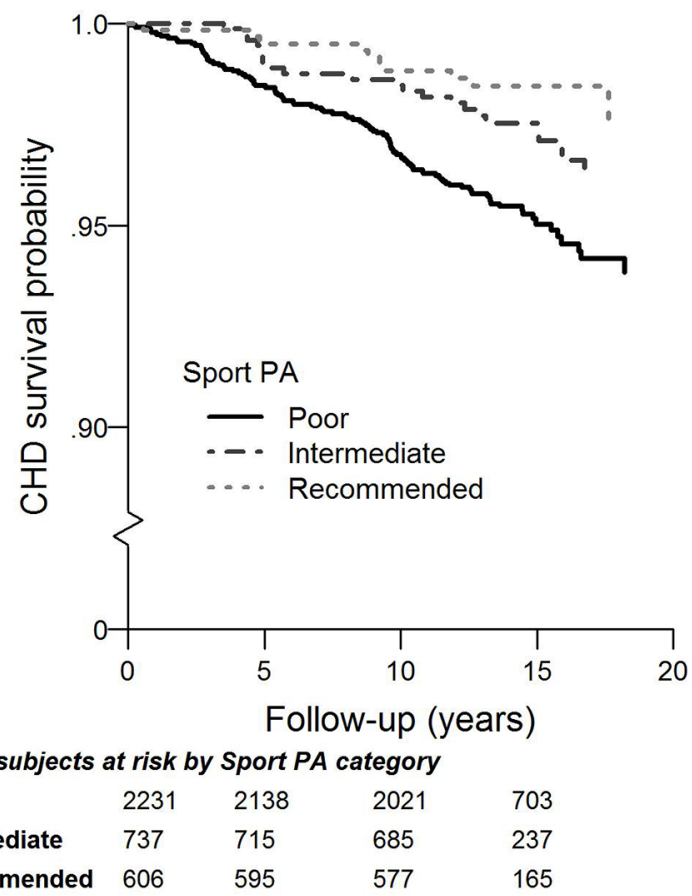

C

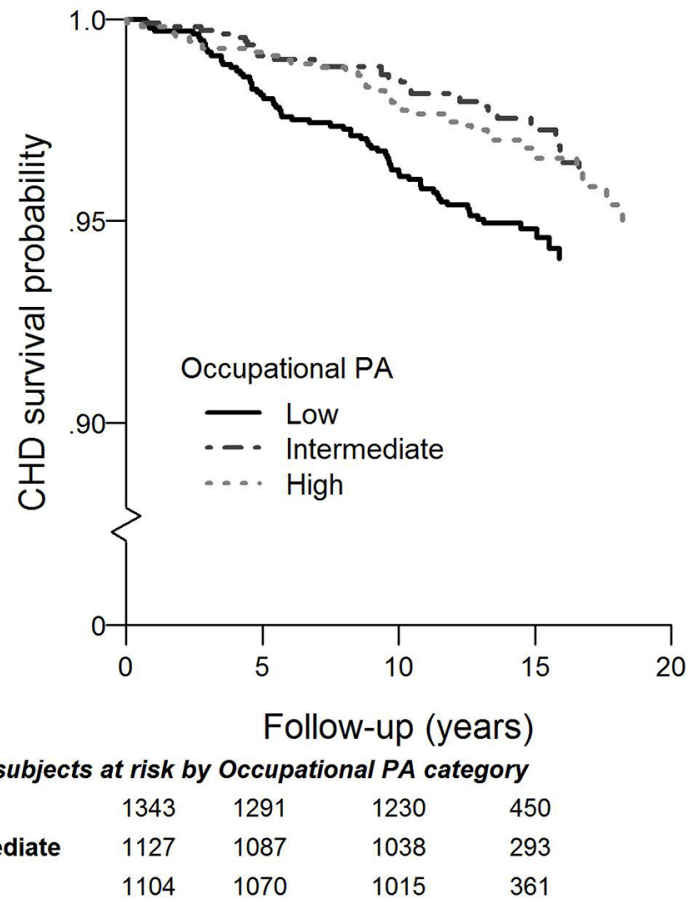

B SpPA and CVD

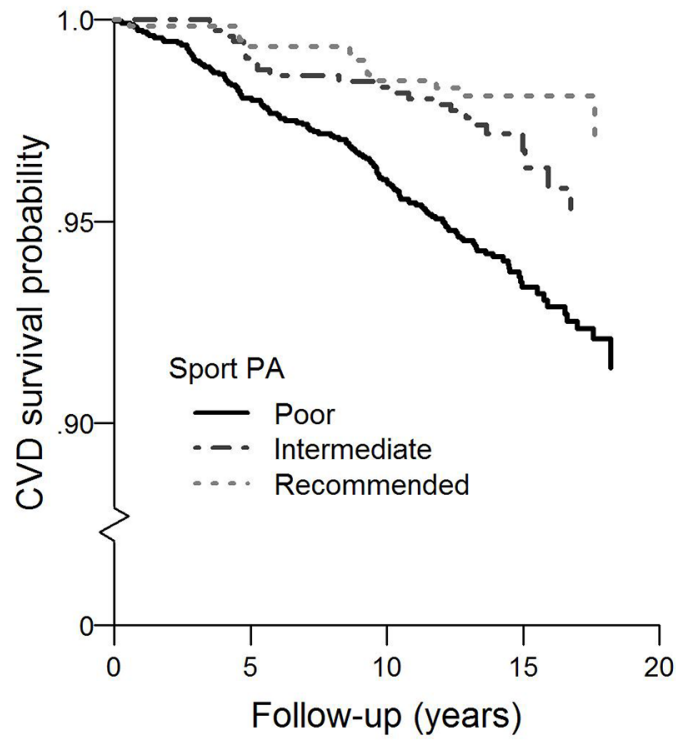

$\begin{array}{llll}2231 & 2132 & 2010 & 690 \\ 737 & 714 & 684 & 234 \\ 606 & 594 & 575 & 164\end{array}$

D OPA and CVD

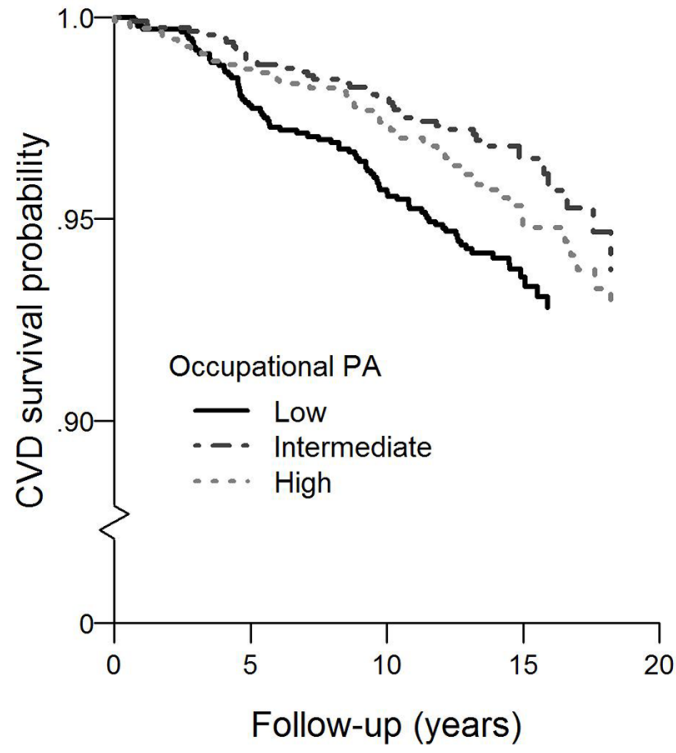

$\begin{array}{llll}1343 & 1287 & 1223 & 441 \\ 1127 & 1086 & 1035 & 292 \\ 1104 & 1067 & 1011 & 353\end{array}$

Figure 1 Kaplan-Meyer survival curves for SpPA (top) and OPA (bottom) categories, for CHD (left side) and CVD (right side) endpoints. CHD, coronary heart disease; CVD, cardiovascular disease; OPA, occupational physical activity; SpPA, sport physical activity.

CVD risks in adult North Italian working men. These reductions, when intermediate and recommended levels of sport PA were combined, mounted to $28 \%$ for CHD and $32 \%$ for CVD, the latter being statistically significant. Similar results in men were reported by most of the previous primary and secondary studies for LTPA. ${ }^{10} 1417182122$ For women, the results are less consistent, with a reduced CHD risk in a Finnish study, ${ }^{15}$ and with mostly null effects reported by another study. ${ }^{18}$ Furthermore, the protective effects in our study were only partially reduced when adjusting for behavioural and metabolic risk factors. In our data, lower prevalence of BMI and current cigarette smokers and means of total cholesterol with higher HDL cholesterol levels were found among those performing recommended levels of SpPA, indicating potential mediation effects of these factors. Other studies adjusted for different sets of demographic characteristics and CVD risk factors, ${ }^{14}$ and this might explain in part the heterogeneity of their results. 
Table 4 Risk factor-adjusted HRs (with 95\% Cls) for SpPA in different OPA categories, and test for SpPA-OPA interaction on the incidence of CHD and CVD events

\begin{tabular}{|c|c|c|c|c|c|c|}
\hline \multirow[b]{2}{*}{ OPA } & \multirow[b]{2}{*}{ SpPA } & \multirow[b]{2}{*}{$\mathrm{N}$} & \multicolumn{2}{|c|}{ CHD events $(n=135)$} & \multicolumn{2}{|c|}{ CVD events $(n=174)$} \\
\hline & & & Rate & HR $(95 \% \mathrm{CI})$ & Rate & $\mathrm{HR}(95 \% \mathrm{Cl})$ \\
\hline Low & Poor & 799 & 4.09 & REF & 4.79 & REF \\
\hline Low & Intermediate/recommended & 544 & 1.56 & $0.45(0.24$ to 0.87$)$ & 1.86 & $0.45(0.25$ to 0.82$)$ \\
\hline Intermediate & Poor & 673 & 2.01 & REF & 2.98 & REF \\
\hline Intermediate & Intermediate/recommended & 454 & 2.29 & $1.20(0.54$ to 2.67$)$ & 2.41 & $0.93(0.43$ to 1.98$)$ \\
\hline High & Poor & 759 & 2.40 & REF & 3.57 & REF \\
\hline High & Intermediate/recommended & 345 & 3.56 & $1.84(0.88$ to 3.87$)$ & 4.98 & $1.66(0.87$ to 3.14$)$ \\
\hline \multicolumn{2}{|c|}{ Heterogeneity test $\mathrm{P}$ value* } & - & - & 0.02 & - & 0.01 \\
\hline \multicolumn{3}{|c|}{ Interaction test $P$ value $\ddagger^{\circ}$} & - & 0.02 & - & 0.01 \\
\hline
\end{tabular}

Men aged 25-64 years, free of CVD and currently employed at baseline ( $\mathrm{n}=3574)$. Rates (x1000 person-years) are age adjusted and estimated at the sample mean age.

Adjusted for age, cohort type (population-based vs factory-based), educational level, body mass index, total cholesterol, HDL cholesterol, systolic BP, current smoking, diabetes

and alcohol intake.

${ }^{*}$ Wald $\chi^{2}$ test $(5 \mathrm{df})$.

tWald $\chi^{2}$ test for interaction (2 df).

BP, blood pressure; CHD, coronary heart disease; CVD, cardiovascular disease; HDL, high-density lipoprotein; OPA, occupational physical activity; SpPA, sport physical activity.

We focused on three categories of SpPA, based on AHA recommended levels, ${ }^{3}{ }^{4}$ which can be derived from the Baecke Questionnaire, and are well characterised in terms of frequency, minutes per week and intensity. Most of other papers used LTPA, which includes a combination of sport and recreational (walking and bicycling during leisure time and for going to work) domains of PA..$^{15} 18$ A few more recent studies better characterised PA in terms of frequency (times per week) and duration (minutes per session). ${ }^{17} 22$ The variety of LTPA definitions may explain in part the differences in estimating the size of the effects, as PA levels may have different meanings in different geographical, gender and age groups, ${ }^{10}$ and a non-quantitative scoring may not grasp such differences. The Baecke Questionnaire includes items exploring the recreational domain of LTPA. In our data, these items showed a poor internal correlation (Cronbach $\alpha$ coefficient $<0.4$ ), modest predictive ability (dissimilar single itemendpoints associations) and reduced construct validity (only the item 'During leisure time I walk' loads the factor with an acceptable coefficient of 0.72 ), indicating that walking was the single, main determinant of the LPTA score (data not shown). Due to this poor performance of these items, we excluded recreational LTPA from our analysis.

Our study found a statistically significant $66 \%$ increase in CHD incidence rates among workers with low levels of OPA, when compared with the intermediate OPA category and adjusting for SpPA as well as for other major CVD risk factors. We also estimated a $18 \%$ increase in both CHD and CVD risks among workers with higher levels of OPA, neither statistically significant. A recent meta-analysis ${ }^{12}$ reported similar results for the higher but not for the lower levels of OPA. The discrepancies may be explained by the inclusion of different proportion of sedentary workers in the examined samples, as well as by the conjunct consideration of sport/LTPA (as discussed later in this paper). The higher CVD risk in sedentary people (mainly due to the work component) was also found in other studies. ${ }^{911}$ On the other hand, a Danish study ${ }^{21}$ did not find a higher risk in ischaemic heart disease mortality in sedentary work activities, but the different endpoint and the effect of Myocardial Infarction (MI) survival should be acknowledged. A Swedish study ${ }^{19}$ did not find an increase in risk of myocardial infarction in high OPA. Again, reasons for these discrepancies may be due to the different methods adopted for the assessment of OPA, ${ }^{10} 14$ and also to the differences in the length of follow-up, as can be seen in figure 1, where OPA differences in risk start to show up after 3-5 years of follow-up.

In our study, the protective effect of AHA recommended and intermediate SpPA levels was strongly dependent of OPA level, with a prominent risk reduction among sedentary workers (55\%), and an opposite relationship for workers with higher level of OPA increasing the risk up to $66 \%$ for CVD and $84 \%$ for CHD. These findings suggest that the strongest effect of SpPA can be detected among people involved in mainly sedentary jobs, in particular when recommended SpPA levels are met and when commuting PA is limited (online supplementary tables S1 and S2). Since these jobs mainly characterise white collars, the residual confounding of social class should be acknowledged, although we adjusted for the educational level. On the other hand, performing heavy physical working tasks and engaging in high levels of sport activities during leisure time may lead to premature exhaustion and fatigue, and generate an overloading of the cardiovascular system, which over years with insufficient recovery can lead to CVD. ${ }^{29}$ Our online supplementary material findings on workers meeting recommended SpPA levels and with intense daily commuting PA strengthen our confidence to the main results, and provide further support to the overloading hypothesis. Moreover, this opposite effect of high SpPA among people with high levels of OPA may be a further explanation of the paradoxical adverse effect of OPA on CVD risk. In table 5, we report the effect of high versus low LTPA/SpPA in different OPA categories from published studies which addressed the interplay between OPA and LTPA/SpPA in working men. One study ${ }^{19}$ was not included as published results do not allow deriving comparable calculations. A Belgian study, ${ }^{22}$ which adopted similar OPA assessment tools and endpoint (CHD incidence), did find the same results. Our study just extends these findings on a longer follow-up period. A Finnish ${ }^{15}$ and an Israeli ${ }^{17}$ study found a prominent protective effect of LTPA in low OPA categories only. Conversely, another Danish study, which used fatal ischaemic heart diseases only as the endpoint, found protective effects of LTPA over all OPA categories. ${ }^{21}$ Finally, Wang et $a l^{20}$ measured OPA adopting the relative aerobic strain or workload method, which takes into account both the absolute energy expenditure of the tasks and the workers' individual aerobic capacity. In this study, the effect of LTPA was modest both in low and high OPA categories. Moreover, a recent report has shown that the beneficial effect of LTPA increasing heart rate variability reduces when 
Table 5 Age and multiple risk factor-adjusted HRs (with 95\% Cl) for incident CHD events on the effect of LTPA within occupational PA categories, published cohort studies, 2007-2016

\begin{tabular}{|c|c|c|c|c|c|c|c|c|c|}
\hline \multirow[b]{2}{*}{ First author, year } & \multirow[b]{2}{*}{ Country } & \multirow[b]{2}{*}{ Endpoint } & \multirow{2}{*}{$\begin{array}{l}\text { Length of follow- } \\
\text { up (years) }\end{array}$} & \multirow{2}{*}{$\begin{array}{l}\text { Gender, } \\
\text { age (years) }\end{array}$} & \multirow{2}{*}{$\begin{array}{l}\text { No. of subjects, } \\
\text { events }\end{array}$} & \multirow{2}{*}{$\begin{array}{l}\text { Sport-LTPA } \\
\text { levels }\end{array}$} & \multicolumn{3}{|l|}{ Occupational PA } \\
\hline & & & & & & & Low & Intermediate & High \\
\hline & & & & $\begin{array}{l}\text { Men, } \\
25-64\end{array}$ & 22877,3073 & & $\begin{array}{l}0.77 \\
(0.67 \text { to } 0.89)^{*}\end{array}$ & NP & $1.04^{*} \dagger$ \\
\hline $\mathrm{Hu}, 2007^{15}$ & Finland & $\mathrm{CHD}, \mathrm{F}+\mathrm{NF}$ & 18.9 & $\begin{array}{l}\text { Women, } \\
\text { 25-64 }\end{array}$ & 24963,1587 & $\begin{array}{l}\text { Moderate to } \\
\text { high vs low }\end{array}$ & $\begin{array}{l}0.77 \\
(0.66 \text { to } 0.91)^{*}\end{array}$ & NP & $0.79^{*} \dagger$ \\
\hline Holtermann, $2009^{21}$ & Denmark & IHD, F & 30 & $\begin{array}{l}\text { Men, } \\
\text { 40-59 }\end{array}$ & 4952,591 & $\begin{array}{l}\text { Moderate to } \\
\text { high vs low }\end{array}$ & $\begin{array}{l}0.70 \\
(0.47 \text { to } 1.07) \ddagger\end{array}$ & $\begin{array}{l}0.75 \\
(0.56 \text { to } 1.0) \ddagger\end{array}$ & $\begin{array}{l}0.65 \\
(0.42 \text { to } 1.0) \ddagger\end{array}$ \\
\hline Clays, $2013^{22}$ & Belgium & $\mathrm{CHD}, \mathrm{F}+\mathrm{NF}$ & 3.15 & $\begin{array}{l}\text { Men, } \\
35-59\end{array}$ & 14337,87 & $\begin{array}{l}\text { Moderate to } \\
\text { high vs low }\end{array}$ & $\begin{array}{l}0.52 \\
(0.26 \text { to } 1.04) \S\end{array}$ & NP & $\begin{array}{l}2.3 \\
(0.75 \text { to } 7.09) \S\end{array}$ \\
\hline Harari, $2015^{17}$ & Israel & $\mathrm{CHD}, \mathrm{F}$ & 22 & $\begin{array}{l}\text { Men, } \\
20-70\end{array}$ & 4819,170 & Yes vs no & $0.56+9$ & NP & $1.0+9$ \\
\hline \multirow[t]{2}{*}{ Wang, $2016^{20}$} & Finland & $\mathrm{AMI}, \mathrm{F}+\mathrm{NF}$ & 20 & $\begin{array}{l}\text { Men, } \\
42-60\end{array}$ & 1891,495 & High vs low & $\begin{array}{l}0.95 \\
(0.73 \text { to } 1.25)^{* *}\end{array}$ & NP & $\begin{array}{l}0.83 \\
(0.57 \text { to } 1.21)^{* *}\end{array}$ \\
\hline & & $\mathrm{CHD}, \mathrm{F}+\mathrm{NF}$ & & & 3574,135 & & $\begin{array}{l}0.45 \\
(0.24 \text { to } 0.87)+t \S\end{array}$ & $\begin{array}{l}1.20 \\
(0.54 \text { to } 2.67)+\dagger\end{array}$ & $\begin{array}{l}1.84 \\
(0.88 \text { to } 3.87)+\dagger\end{array}$ \\
\hline Ferrario, present paper & Italy & $C V D, F+N F$ & 14.2 & $\begin{array}{l}\text { Men, } \\
25-64\end{array}$ & 3574,174 & $\begin{array}{l}\text { Moderate to } \\
\text { high vs low }\end{array}$ & $\begin{array}{l}0.45 \\
(0.25 \text { to } 0.82)+\dagger\end{array}$ & $\begin{array}{l}0.93 \\
(0.43 \text { to } 1.98)+t\end{array}$ & $\begin{array}{l}1.66 \\
(0.87 \text { to } 3.14)+\dagger\end{array}$ \\
\hline
\end{tabular}

*Adjusted for age, study year, education, alcohol, smoking, BMI, systolic BP, cholesterol and history of diabetes. HRs excluding PA due to commuting.

tHRs computed from the reported values in the original tables. Cls are not estimable from the original tables.

$\ddagger$ Adjusted for age, BMI, systolic BP, diastolic BP, treatment of diabetes or hypertension, alcohol, smoking and occupational classes.

$\S$ Adjusted for age, educational level, occupational class, job strain, BMI, smoking, alcohol, diabetes, systolic BP, total cholesterol and HDL cholesterol.

IAdjusted for age, socioeconomic status (number of people/room), educational status, father's country of origin, BMI, total cholesterol, HDL cholesterol, hypertension, diabetes, smoking,

coffee consumption, alcohol, diet and shift work. HRs for 'low' and 'high' OPA refer to the original 'none-mild' and 'moderate-hard' OPA categories (Table 3 of the reference).

${ }^{* *}$ Adjusted for age, education, participation in an unrelated clinical trial, smoking, alcohol, mental strain at work, social support at work and stress from work deadlines.

††Adjusted for age, cohort, educational class, BMI, total cholesterol, HDL cholesterol, systolic BP, current smoking, diabetes and alcohol intake.

AMI, acute myocardial infarction; BMI, body mass index; BP, blood pressure; CHD, coronary heart disease; CVD, cardiovascular disease; F, Fatal; HDL, high-density lipoprotein; IHD, ischaemic

heart disease; LTPA, leisure time physical activity; NF, non fatal; NP, data not present; PA, physical activity.

combined with higher OPA levels. ${ }^{30}$ Anyhow, more collaborative studies are needed to investigate the effects of LTPA/SpPA among workers with high OPA, adopting common and standardised methodologies.

\section{Key messages}

\section{What is already known on this subject?}

While consistent evidence indicates that elevated and intermediate levels of leisure time physical activity decrease the risk of cardiovascular diseases (CVD), the relationship between occupational physical activity (OPA) levels and CVD has showed contrasting results.

\section{What might this study add?}

- Our study, conducted on North Italian male working cohorts, confirms the protective effect of both American Heart Association recommended and intermediate levels of sport activities, with a reduced CVD risk of $32 \%$, as well as a paradoxical effect of higher CVD risks in low and high levels of OPA when compared with the intermediate level.

- The protective effect of recommended and intermediate sport physical activity (SpPA) levels strongly depends on OPA levels, with a prominent risk reduction among sedentary workers ( $55 \%)$, and an opposite relationship for workers with higher level of OPA increasing the risk up to $66 \%$ for CVD and $84 \%$ for coronary heart disease.

\section{How might this impact on clinical practice?}

The increasing number of sedentary workers in postindustrialised societies asks for specific interventions to promote SpPA even at the workplace. If our results were confirmed in larger studies, the CVD prevention recommendation of SpPA ought to consider the level of OPA.
Our study presents some limitations. First, PA was calculated using a self-administered questionnaire, once on the entire follow-up period. We can thus assume some bias due to misclassifications of the exposures: in general, HRs of higher levels of both sport PA and OPA may be underestimated as people getting old moderate the former and are reallocated at work and reduce strenuous tasks. Although we adjusted for major risk factors, we do not have data on nutrition as a potential confounder. Another limitation is the focus on men only, as the low number of events among women determined very unstable effect estimates, in particular for the OPA-SpPA interaction analyses. As mentioned, results in men and women can be heterogeneous because of both different levels of PA and different associations between PA domains and the CVD endpoint, so combining the two gender groups may be misleading and can produce confused findings. Finally, our data do not allow to further investigate the separate effects of walking and lifting at work on the CVD risk, as the large majority of high OPA workers (67\%) reported both frequent walking and lifting.

In conclusion, our study conducted on North Italian male workers suggests a prominent protective effect of AHA recommended and intermediate sport PA levels on CVDs among sedentary workers, and an opposite effect in people involved in physically demanding working tasks. The second finding particularly needs further investigations in other cohorts to assess consistency of the results. Due to the increasing number of sedentary workers in postindustrialised countries, interventions to promote SpPA in working populations could be an important factor to reduce the burden of CVDs. In addition, future CVD prevention guidelines ${ }^{4}$ should consider tailoring the recommendations on the amount of PA during leisure time to the levels of PA at work.

\section{Author affiliations}

${ }^{1}$ Department of Medicine and Surgery, School of Medicine, Research Center in Epidemiology and Preventive Medicine, University of Insubria, Varese, Italy ${ }^{2}$ Occupational Medicine Unit, University hospital of Varese, Varese, Italy 
${ }^{3}$ School of Occupational Medicine, University of Insubria, Varese, Italy

${ }^{4}$ The National Research Centre for the Working Environment, Copenhagen, Denmark

${ }^{5}$ Department of Sports Science and Clinical Biomechanics, University of Southern Denmark, Odense, Denmark

${ }^{6}$ Department of Public Health, Ghent University, Ghent, Belgium

${ }^{7}$ Department of Medicine and Surgery, University of Milano-Bicocca, Monza, Italy

${ }^{8}$ IRCCS Multimedica, Sesto San Giovanni, Italy

Collaborators The Cohorts Collaborative Study in Northern Italy (CCSNI) Research Group: MONICA-Brianza cohorts: PIs: Professor Marco Mario Ferrario, Professor Giancarlo Cesana; Baseline data collection: Professor R Sega, Dr M T Gussoni, Dr F Duzioni, Sig L Bolognesi; Data quality assessment: Dr G Veronesi, Dr C Fornari, Dr L Bertù. PAMELA study: PI: Professor Guido Grassi. Professor R Sega and Professor G Mancia (former PIs). Baseline data collection and follow-up activities: Professor R Sega, Dr M Bombelli. Data quality assessment: Dr R Facchetti. SEMM Study: PI: Professor Marco M Ferrario. Baseline data collection: Dr A Vivaldi. Data quality assessment: Dr G Veronesi, Dr C Fornari, Dr L Bertù. Laboratory and biobanking: Professor P Brambilla, Dr S Signorini.

Contributors MMF originally conceived the research question. MMF, MR and GV drafted the manuscript and MMF and GV prepared the revisions. MMF is the PI of the SEMM study. MMF and GC are co-PIs of the MONICA Brianza cohorts and GG is $\mathrm{PI}$ for the PAMELA study. GV was responsible for the statistical analyses. AH, MC, EC, $\mathrm{RB}, \mathrm{GG}$ and $\mathrm{GC}$ critically revised the text, with particular reference to the introduction and to the discussion sections.

Funding This work and the latest activities of the Brianza, PAMELA and SEMM cohorts have been funded by the Health Administration of the Lombardia Region (grants nos. 17155/2004 and 10800/2009). The follow-up was partially supported with grants from the Italian Ministry of Health (grant 2012/597) and it was carried out in collaboration with the Department for Cardiovascular Dysmetabolic and Ageing -Associated Diseases of the Istituto Superiore di Sanità in Rome.

Competing interests None declared.

Ethics approval The MONICA-Brianza and the PAMELA studies were approved by the Ethical Committee of the University Hospital of Monza (Italy). The SEMM study was approved by the Ethical Committee of the University Hospital of Varese (Italy)

Provenance and peer review Not commissioned; externally peer reviewed.

(c) Article author(s) (or their employer(s) unless otherwise stated in the text of the article) 2018. All rights reserved. No commercial use is permitted unless otherwise expressly granted.

\section{REFERENCES}

1 GBD 2015 Mortality and Causes of Death Collaborators. Global, regional, and national life expectancy, all-cause mortality, and cause-specific mortality for 249 causes of death, 1980-2015: a systematic analysis for the Global Burden of Disease Study 2015. Lancet 2016:388:1459-544.

2 Townsend N, Wilson L, Bhatnagar P, et al. Cardiovascular disease in Europe: epidemiological update 2016. Eur Heart J 2016:37:3232-45.

3 US Department of Health and Human Services. Physical activity guidelines for Americans. Washington, DC: U.S. Department of Health and Human Services, 2008.

4 Piepoli MF, Hoes AW, Agewall S, et al. 2016 European Guidelines on cardiovascular disease prevention in clinical practice: The Sixth Joint Task Force of the European Society of Cardiology and Other Societies on Cardiovascular Disease Prevention in Clinical Practice (constituted by representatives of 10 societies and by invited experts)Developed with the special contribution of the European Association for Cardiovascular Prevention \& Rehabilitation (EACPR). Eur Heart J 2016;37:2315-81.

5 World Health Organization. Global recommendations on physical activity for health. Geneva: World Health Organization, 2010.

6 Lloyd-Jones DM, Hong Y, Labarthe D, et al. Defining and setting national goals for cardiovascular health promotion and disease reduction: the American Heart Association's strategic Impact Goal through 2020 and beyond. Circulation 2010;121:586-613.

7 Sofi F, Capalbo A, Cesari F, et al. Physical activity during leisure time and primary prevention of coronary heart disease: an updated meta-analysis of cohort studies. Eur J Cardiovasc Prev Rehabil 2008;15:247-57.
8 Nocon M, Hiemann T, Müller-Riemenschneider F, et al. Association of physical activity with all-cause and cardiovascular mortality: a systematic review and meta-analysis. Eur J Cardiovasc Prev Rehabil 2008;15:239-46.

9 Khaw KT, Jakes R, Bingham S, et al. Work and leisure time physical activity assessed using a simple, pragmatic, validated questionnaire and incident cardiovascular disease and all-cause mortality in men and women: The European Prospective Investigation into Cancer in Norfolk prospective population study. Int J Epidemiol 2006;35:1034-43.

10 Bell EJ, Lutsey PL, Windham BG, et al. Physical activity and cardiovascular disease in African americans in ARIC. Med Sci Sports Exerc 2013;45:901-7.

11 Cheng X, Li W, Guo J, et al. Physical activity levels, sport activities, and risk of acute myocardial infarction: results of the INTERHEART study in China. Angiology 2014;65:113-21.

12 Li J, Loerbroks A, Angerer P. Physical activity and risk of cardiovascular disease: what does the new epidemiological evidence show? Curr Opin Cardiol 2013;28:575-83.

13 Autenrieth CS, Baumert J, Baumeister SE, et al. Association between domains of physical activity and all-cause, cardiovascular and cancer mortality. Eur J Epidemiol 2011:26:91-9.

14 Li J, Siegrist J. Physical activity and risk of cardiovascular disease--a meta-analysis of prospective cohort studies. Int J Environ Res Public Health 2012;9:391-407.

15 Hu G, Jousilahti P, Borodulin K, et al. Occupational, commuting and leisure-time physical activity in relation to coronary heart disease among middle-aged Finnish men and women. Atherosclerosis 2007:194:490-7.

$16 \mathrm{Hu} \mathrm{G}$, Sarti C, Jousilahti P, et al. Leisure time, occupational, and commuting physical activity and the risk of stroke. Stroke 2005:36:1994-9.

17 Harari G, Green MS, Zelber-Sagi S. Combined association of occupational and leisuretime physical activity with all-cause and coronary heart disease mortality among a cohort of men followed-up for 22 years. Occup Environ Med 2015;72:617-24.

$18 \mathrm{Hu} \mathrm{GC}$, Chien KL, Hsieh SF, et al. Occupational versus leisure-time physical activity in reducing cardiovascular risks and mortality among ethnic Chinese adults in Taiwan. Asia Pac J Public Health 2014;26:604-13.

19 Johnsen AM, Alfredsson L, Knutsson A, et al. Association between occupational physical activity and myocardial infarction: a prospective cohort study. BMJ Open 2016;6: 012692

20 Wang A, Arah OA, Kauhanen J, et al. Effects of leisure-time and occupational physical activities on 20-year incidence of acute myocardial infarction: mediation and interaction. Scand I Work Environ Health 2016;42:423-34.

21 Holtermann A, Mortensen OS, Burr $\mathrm{H}$, et al. The interplay between physical activity at work and during leisure time--risk of ischemic heart disease and all-cause mortality in middle-aged Caucasian men. Scand J Work Environ Health 2009;35:466-74.

22 Clays E, De Bacquer D, Janssens $\mathrm{H}$, et al. The association between leisure time physical activity and coronary heart disease among men with different physical work demands: a prospective cohort study. Eur J Epidemiol 2013;28:241-7.

23 Ferrario M, Sega R, Cesana G. Lessons from the MONICA study in northern Italy. J Hypertens Supp/ 1991;9:S7-S14.

24 Ferrario MM, Veronesi G, Chambless LE, et al. The contribution of major risk factors and job strain to occupational class differences in coronary heart disease incidence: the MONICA Brianza and PAMELA population-based cohorts. Occup Environ Med 2011;68:717-22.

25 Ferrario M, Chiodini P, Chambless LE, et al. Prediction of coronary events in a low incidence population. Assessing accuracy of the CUORE Cohort Study prediction equation. Int J Epidemiol 2005;34:413-21.

26 Baecke JA, Burema J, Frijters JE. A short questionnaire for the measurement of habitual physical activity in epidemiological studies. Am J Clin Nutr 1982;36:936-42.

27 Jacobs DR, Ainsworth BE, Hartman TJ, et al. A simultaneous evaluation of 10 commonly used physical activity questionnaires. Med Sci Sports Exerc 1993;25:81-91.

28 Strath SJ, Kaminsky LA, Ainsworth BE, et al. Guide to the assessment of physical activity: Clinical and research applications: a scientific statement from the American Heart Association. Circulation 2013:128:2259-79.

29 Holtermann A, Krause N, van der Beek AJ, et al. The physical activity paradox: six reasons why occupational physical activity (OPA) does not confer the cardiovascular health benefits that leisure time physical activity does. Br I Sports Med 2017. doi: 10.1136/bjsports-2017-097965. Epub ahead of print 10 Aug 2017.

30 Hallman DM, Birk Jørgensen M, Holtermann A. On the health paradox of occupational and leisure-time physical activity using objective measurements: Effects on autonomic imbalance. PLoS One 2017:12:e0177042. 